\title{
Exact solutions and conservation laws for the modified equal width-Burgers equation
}

https://doi.org/10.1515/phys-2018-0099

Received May 31, 2018; accepted July 2, 2018

\begin{abstract}
In this paper we study the modified equal widthBurgers equation, which describes long wave propagation in nonlinear media with dispersion and dissipation. Using the Lie symmetry method in conjunction with the $\left(G^{\prime} / G\right)$ expansion method we construct its travelling wave solutions. Also, we determine the conservation laws by invoking the new conservation theorem due to Ibragimov. As a result we obtain energy and linear momentum conservation laws.
\end{abstract}

Keywords: Modified equal width-Burgers equation; Lie point symmetries; exact solutions; conservation laws

PACS: 02.30.Jr, 04.20.Jb, 11.30.-j

\section{Introduction}

It is well-known that majority of the real-world physical phenomena are modeled by mathematical equations, especially nonlinear partial differential equations (NLPDEs). These phenomena include the problems from fluid mechanics, elasticity, plasma and optical fibers, relativity, gas dynamics, thermodynamics, and many more. In order to comprehend the understanding of such physical phenomena it is vital to look for exact solutions of the NLPDEs. During the last sixty years many scientists and mathematicians developed several effective and useful methods for obtaining exact solutions of NLPDEs. These

\footnotetext{
^Corresponding Author: Chaudry Masood Khalique: International Institute for Symmetry Analysis and Mathematical Modelling, Department of Mathematical Sciences, North-West University, Mafikeng Campus, Private Bag X 2046, Mmabatho 2735, Republic of South Africa,

E-mail: Masood.Khalique@nwu.ac.za

Innocent Simbanefayi: International Institute for Symmetry Analysis and Mathematical Modelling, Department of Mathematical Sciences, North-West University, Mafikeng Campus, Private Bag X 2046, Mmabatho 2735, Republic of South Africa,

E-mail: isimbanefayi@yahoo.com
}

include the simplified Hirota's method [1, 2], the tanhcoth method [3], the sine-cosine method [4], the simplest equation method[5], the homogeneous balance method [6], the inverse scattering transform method [7], Hirota's bilinear method [8], the $\left(G^{\prime} / G\right)$-expansion method [9, 10], Riccati-Bernoulli sub-ordinary differential equation method [11], Jacobi elliptic function expansion method[12, 13], Kudryashov method [14, 15], the Lie symmetry method [16-21], just to mention a few.

On the other hand conservation laws are very important in the study of NLPDEs and much research has been done on different methods of obtaining conservation laws. Conservation laws are essential in determining the extent of integrability of differential equations, development of numerical schemes, reduction and solutions of partial differential equations. See, for example [22-29] and references therein.

The Burgers equation

$$
u_{t}+u u_{x}-v u_{x x}=0
$$

where $v$ is a constant defining the kinematic viscosity models the turbulent flow in a channel and describes the effect of coupling between diffusion and convection processes on a fluid. It first appeared in academic circles in [30]. Over the years, various researchers have applied modifications to (1.1) and used many different methods to study the equation. For example, the equal width equation

$$
u_{t}-u u_{x}-u_{t x x}=0
$$

was first introduced in [31]. It describes amongst others, nonlinear dispersive waves such as shallow water waves and nonlinear waves in plasmas. Extensive work was done on Equation (1.2) in constructing numerical solutions [32, 33]. Arora et al. [34] performed the reduced differential transform method to find the numerical solution of the equal width wave equation and the exact analytical solution of the inviscid Burgers equation with initial conditions.

The modified equal width equation

$$
u_{t}+a u^{2} u_{x}-b u_{t x x}=0
$$

was studied in [35] and solitary wave solutions were obtained. Furthermore, using Quintic B-spline method the 
interactions through computer simulation were observed. In [36] the sine-cosine and the tanh methods were employed to obtain exact solutions of (1.3) and two of its variants, which included compactons, solitons, solitary patterns, and periodic solutions. Hasan [37] presented the numerical solution for (1.3) using Fourier spectral method that discretizes the space variable and Leap-frog method scheme for time dependence.

The generalised equal width equation

$$
u_{t}+a u^{m} u_{x}-b u_{t x x}=0
$$

was studied in [38] and its solitary wave solutions were obtained by a collocation method using quadratic B-spline at the midpoints.

In [39] the author introduced the modified equal width-Burgers (MEW-Burgers) equation

$$
u_{t}+\alpha u^{2} u_{x}+\omega u_{x x}-\beta u_{t x x}=0,
$$

which describes long wave propagation in nonlinear media with dispersion and dissipation. Here $\alpha, \beta$ are positive parameters and $\omega$ is a damping parameter. The bifurcation behaviour and an external periodic perturbation of the MEW-Burgers equation (1.5) was studied [39].

In this paper using the Lie symmetry method and the $\left(G^{\prime} / G\right)$-expansion method we construct travelling wave solutions of (1.5). Furthermore, we derive the conservation laws by applying the new conservation theorem due to Ibragimov.

\section{Travelling wave solutions of (1.5)}

In this section we obtain travelling wave solutions of (1.5) by employing Lie symmetry analysis together with the $\left(G^{\prime} / G\right)$-expansion method.

\subsection{Lie point symmetries and reduction of} (1.5)

We begin by determining the Lie point symmetries of (1.5). The vector field

$$
X=\xi^{1}(t, x, u) \frac{\partial}{\partial t}+\xi^{2}(t, x, u) \frac{\partial}{\partial x}+\eta(t, x, u) \frac{\partial}{\partial u}
$$

is a Lie point symmetry of (1.5) if and only if

$$
\left.X^{[3]}\left(u_{t}+\alpha u^{2} u_{x}+\omega u_{x x}-\beta u_{t x x}\right)\right|_{(1.5)}=0,
$$

where

$$
X^{[3]}=X+\zeta_{1} \frac{\partial}{\partial u_{t}}+\zeta_{2} \frac{\partial}{\partial u_{x}}+\zeta_{22} \frac{\partial}{\partial u_{x x}}+\zeta_{122} \frac{\partial}{\partial u_{t x x}}
$$

is the third prolongation of $X$ [18].

Expanding (2.7), we obtain

$$
\begin{aligned}
& \eta_{t}+2 u_{x} u_{t} \xi_{u}^{2}+2 \beta u_{t t x} \xi_{t}^{1}-2 \mu u_{t, x} \xi_{x}^{1}+2 \beta u_{t x}^{2} \xi_{u}^{1}+\beta u_{t x} \xi_{x x}^{2} \\
& -\mu u_{x} \xi_{x x}^{2}+\beta u_{t t} \xi_{x x}^{1}+2 \mu u_{x} \eta_{x u}-\mu u_{t} \xi_{x x}^{1}-2 \beta u_{t, x} \eta_{x u} \\
& +\alpha u^{2} \eta_{x}+\beta \xi_{t x x}^{1} u_{t}+\beta \xi_{t x x}^{2} u_{x}+2 \beta \xi_{t x}^{1} u_{t, x}+2 \beta u_{x x} \xi_{t x}^{2} \\
& -\beta u_{t} \eta_{x x u}-\beta \eta_{t u u} u_{x}^{2}-\beta \eta_{t u} u_{x x}+\beta \xi_{x x u}^{1} u_{t}^{2}+u_{x}^{3} \beta \xi_{t u u}^{2} \\
& +2 \beta \xi_{t x u}^{2} u_{x}^{2}-2 \beta u_{x} \eta_{t x u}-2 \mu u_{x}^{2} \xi_{x u}^{2}-\mu u_{x}^{3} \xi_{u u}^{2}+\mu u_{x}^{2} \eta_{u u} \\
& +\mu u_{x x} \xi_{t}^{1}+\beta u_{x x x} \xi_{t}^{2}+2 \xi_{x}^{2} u_{t}-\xi_{t}^{2} u_{x}+\xi_{u}^{1} u_{t}^{2}+\mu \eta_{x x}-\beta \eta_{t x x} \\
& +\beta u_{t} u_{x} \xi_{u x x}^{2}+3 \beta u_{x} u_{x x} \xi_{t u}^{2}+\beta u_{t} u_{x}^{2} \xi_{t u u}^{1}+2 \beta u_{t} u_{x} \xi_{t x u}^{1} \\
& -\beta u_{t} u_{x}^{2} \eta_{u u u}+2 \alpha u u_{x} \eta+\beta u_{t}^{2} u_{x}^{2} \xi_{u u u}^{1}+\beta u_{x x} u_{t} \xi_{t u}^{1} \\
& +2 u_{x} u_{t x} \beta \xi_{t u}^{1}+4 \beta u_{t} u_{t x} \xi_{x u}^{1}+2 \beta u_{x} u_{t t} \xi_{x u}^{1}-2 \mu u_{t} u_{x} \xi_{x u}^{1} \\
& +2 u_{x x} u_{t} \beta \xi_{x u}^{2}+4 u_{x} \beta u_{t x} \xi_{x u}^{2}+3 \beta u_{x}^{2} u_{t x} \xi_{u u}^{2}-\beta u_{x x} u_{t} \eta_{u u} \\
& -2 \beta u_{t x} \eta_{u u}+\beta u_{t}^{2} u_{x x} \xi_{u u}^{1}+\beta u_{x}^{2} u_{t t} \xi_{u u}^{1}-\mu u_{t} u_{x}^{2} \xi_{u u}^{1} \\
& +\alpha u^{2} u_{x} \xi_{x}^{2}+\alpha u^{2} u_{x} \xi_{t}^{1}+2 \xi_{u}^{2} \alpha u^{2} u_{x}^{2}+\xi_{u}^{2} \beta u_{t} u_{x x x} \\
& +3 \xi_{u}^{2} \beta u_{t x} u_{x x}-\alpha u^{2} u_{t} \xi_{x}^{1}+2 \beta u_{t t x} u_{x} \xi_{u}^{1}+\beta u_{x x} u_{t t} \xi_{u}^{1} \\
& +\mu u_{x x} u_{t} \xi_{u}^{1}-2 \mu u_{x} u_{t x} \xi_{u}^{1}+u_{t} u_{x}^{3} \beta \xi_{u u u}^{2}+2 \beta u_{t}^{2} u_{x} \xi_{x u u}^{1} \\
& +2 \beta u_{t} u_{x}^{2} \xi_{x u u}^{2}-2 \beta u_{t} u_{x} \eta_{x u u}+3 \beta u_{t} u_{x} u_{x x} \xi_{u u}^{2} \\
& +4 \beta u_{t} u_{x} u_{t x} \xi_{u u}^{1}+\alpha u^{2} u_{t} u_{x} \xi_{u}^{1}=0 .
\end{aligned}
$$

Splitting the above equation with respect to derivatives of $u$ yields the system of ten overdetermined linear partial differential equations

$$
\begin{aligned}
& \xi_{x}^{1}=0, \\
& \xi_{u}^{1}=0, \\
& \xi_{t}^{2}=0 \\
& \xi_{u}^{2}=0, \\
& \eta_{u u}=0, \\
& \xi_{x x}^{2}-2 \eta_{x u}=0, \\
& \mu \xi_{t}^{1}-\beta \eta_{t u}=0, \\
& 2 \xi_{x}^{2}-\beta \eta_{x x u}=0, \\
& \mu \eta_{x x}-\beta \eta_{t x x}+\eta_{t}+\alpha u^{2} \eta_{x}=0, \\
& \alpha u^{2} \xi_{x}^{2}+\alpha u^{2} \xi_{t}^{1}+2 \alpha u \eta+2 \mu \eta_{x u}-\mu \xi_{x x}^{2}-2 \beta \eta_{t x u}=0
\end{aligned}
$$

Solving (2.9)-(2.18) for $\xi^{1}, \xi^{2}$ and $\eta$ we obtain

$$
\xi^{1}=C_{1}, \xi^{2}=C_{2}, \eta=0,
$$

which results in two translation symmetries

$$
X_{1}=\frac{\partial}{\partial t}, X_{2}=\frac{\partial}{\partial x} .
$$

A linear combination of these Lie point symmetries, that is, $X=X_{1}+c X_{2}$ produces the associated Lagrange equations

$$
\frac{d t}{1}=\frac{d x}{c}=\frac{d u}{0},
$$


which upon solving yields the two invariants $z=x-c t$ and $U=u$, and hence the group-invariant solution $u=$ $U(z)$. Taking $U$ and $z$ as the new dependent and independent variables respectively, equation (1.5) is transformed into the third-order nonlinear ordinary differential equation (ODE)

$$
\beta c U^{\prime \prime \prime}(z)-c U^{\prime}(z)+\omega U^{\prime \prime}(z)+\alpha U(z)^{2} U^{\prime}(z)=0 .
$$

\subsection{Solution of (2.20) using $\left(G^{\prime} / G\right)$-expansion method}

In this subsection we use the $\left(G^{\prime} / G\right)$-expansion method [9] to obtain solutions of (2.20). Firstly, we assume that

$$
U(z)=\sum_{i=0}^{m} A_{i}\left(\frac{G^{\prime}(z)}{G(z)}\right)^{i}
$$

is the solution to (2.20), where $A_{0}, A_{1}, \cdots A_{m}$ are to be determined. The balancing procedure is used to find the value of $m$, a positive integer. In our case the balancing procedure yields $m=1$, thus (2.21) becomes

$$
U(z)=A_{0}+A_{1}\left(\frac{G^{\prime}(z)}{G(z)}\right) .
$$

Secondly, we substitute (2.22) into (2.20) and simultaneously use the second-order ODE

$$
G^{\prime \prime}(z)+\lambda G^{\prime}(z)+\mu G(z)=0,
$$

where $\lambda$ and $\mu$ are arbitrary constants. This yields

$$
\begin{aligned}
& A_{1} \lambda \mu \omega+A_{1} c \mu-2 A_{1} \beta c \mu^{2}-\alpha A_{0}^{2} A_{1} \mu-A_{1} \beta c \lambda^{2} \mu \\
& +\left(A_{1} c \lambda-8 A_{1} \beta c \lambda \mu-A_{1} \beta c \lambda^{3}+2 A_{1} \mu \omega+A_{1} \lambda^{2} \omega\right. \\
& \left.-\alpha A_{0}^{2} A_{1} \lambda-2 \alpha A_{0} A_{1}^{2} \mu\right)\left(\frac{G^{\prime}(z)}{G(z)}\right)+\left(A_{1} c-7 A_{1} \beta c \lambda^{2}\right. \\
& \left.-8 A_{1} \beta c \mu-\alpha A_{0}^{2} A_{1}-2 \alpha A_{0} A_{1}^{2} \lambda-\alpha A_{1}^{3} \mu+3 A_{1} \lambda \omega\right) \\
& \left(\frac{G^{\prime}(z)}{G(z)}\right)^{2}+\left(2 A_{1} \omega-2 \alpha A_{0} A_{1}^{2}-\alpha A_{1}^{3} \lambda-12 A_{1} \beta c \lambda\right) \\
& \left(\frac{G^{\prime}(z)}{G(z)}\right)^{3}-\left(6 A_{1} \beta c+\alpha A_{1}^{3}\right)\left(\frac{G^{\prime}(z)}{G(z)}\right)^{4}=0 .
\end{aligned}
$$

Collecting terms with like powers of $\left(G^{\prime} / G\right)$ and equating them to zero gives the overdetermined system of five algebraic equations

$$
\begin{aligned}
& \left(\frac{G^{\prime}(z)}{G(z)}\right)^{4}: \alpha A_{1}^{3}+6 A_{1} \beta c=0, \\
& \left(\frac{G^{\prime}(z)}{G(z)}\right)^{3}: \alpha A_{1}^{3} \lambda+2 \alpha A_{0} A_{1}^{2}+12 A_{1} \beta c \lambda-2 A_{1} \omega=0,
\end{aligned}
$$

$$
\begin{aligned}
\left(\frac{G^{\prime}(z)}{G(z)}\right)^{2}: & 2 \alpha A_{0} A_{1}^{2} \lambda+\alpha A_{1}^{3} \mu+\alpha A_{0}^{2} A_{1}+7 A_{1} \beta c \lambda^{2} \\
& +8 A_{1} \beta c \mu-A_{1} c-3 A_{1} \lambda \omega=0, \\
\left(\frac{G^{\prime}(z)}{G(z)}\right)^{1}: & \alpha A_{0}^{2} A_{1} \lambda+2 \alpha A_{0} A_{1}^{2} \mu+A_{1} \beta c \lambda^{3}+8 A_{1} \beta c \lambda \mu \\
& -A_{1} c \lambda-A_{1} \lambda^{2} \omega-2 A_{1} \mu \omega=0, \\
\left(\frac{G^{\prime}(z)}{G(z)}\right)^{0}: & A_{1} \lambda \mu \omega+A_{1} c \mu-\alpha A_{0}^{2} A_{1} \mu-A_{1} \beta c \lambda^{2} \mu \\
& -2 A_{1} \beta c \mu^{2}=0 .
\end{aligned}
$$

Using Mathematica, two solutions of the above system of algebraic equations are

Solution set 1

$$
A_{0}=\sqrt{\frac{-2 \lambda \omega}{3 \alpha}}, A_{1}=\sqrt{\frac{-6 \omega}{\lambda \alpha}}, \quad c=\frac{2}{3 \lambda}\left(3 \mu-\lambda^{2}\right) .
$$

Solution set 2

$$
A_{0}=-\sqrt{\frac{2 \omega \sqrt{\mu}}{\alpha}}, A_{1}=3 \sqrt{\frac{2 \omega}{\alpha \sqrt{\mu}}}, \quad c=\frac{-4 \omega \sqrt{\mu}}{3} .
$$

Thus corresponding to solution set 1 above, we have the following three types of solutions for the MEW-Burgers (1.5):

Case 1.1 For $M=\lambda^{2}-4 \mu>0$, we obtain the hyperbolic function solution

$$
\begin{aligned}
u(t, x)= & \left(1+\frac{3}{\lambda}\right) \sqrt{\frac{-2 \lambda \omega}{3 \alpha}} \\
& \left(\Delta_{1} \frac{A \cosh \left(\Delta_{1} z\right)+B \sinh \left(\Delta_{1} z\right)}{A \sinh \left(\Delta_{1} z\right)+B \cosh \left(\Delta_{1} z\right)}-\frac{\lambda}{2}\right),
\end{aligned}
$$

where $\Delta_{1}=\sqrt{M} / 2, z=x-2\left(3 \mu-\lambda^{2}\right) t /(3 \lambda)$ and $A$ and $B$ are constants.

Case 1.2 For $M=\lambda^{2}-4 \mu<0$, we obtain the trigonometric function solution

$$
\begin{aligned}
u(t, x)= & \left(1+\frac{3}{\lambda}\right) \sqrt{\frac{-2 \lambda \omega}{3 \alpha}} \\
& \left(\Delta_{2} \frac{A \sin \left(\Delta_{2} z\right)+B \cos \left(\Delta_{2} z\right)}{A \cos \left(\Delta_{2} z\right)+B \sin \left(\Delta_{2} z\right)}-\frac{\lambda}{2}\right),
\end{aligned}
$$

where $\Delta_{2}=\sqrt{-M} / 2, z=x-2\left(3 \mu-\lambda^{2}\right) t /(3 \lambda)$ and $A$ and $B$ are constants.

Case 1.3 For $M=\lambda^{2}-4 \mu=0$, we obtain the rational function solution

$$
u(t, x)=\left(1+\frac{3}{\lambda}\right) \sqrt{\frac{-2 \lambda \omega}{3 \alpha}}\left(\frac{B}{B z+A}-\frac{\lambda}{2}\right),
$$

where $z=x-2\left(3 \mu-\lambda^{2}\right) t /(3 \lambda)$ and $A$ and $B$ are constants.

Similarly, considering the solution set 2 we obtain the following three types of solutions for the MEW-Burgers (1.5): 
Case 2.1 For $M=\lambda^{2}-4 \mu>0$, we obtain the hyperbolic function solution

$$
\begin{aligned}
u(t, x)= & (1+4 \beta \lambda) \sqrt{\frac{2 \sqrt{\mu} \omega}{\alpha}} \\
& \left(\frac{\lambda}{2}-\Delta_{1} \frac{A \cosh \left(\Delta_{1} z\right)+B \sinh \left(\Delta_{1} z\right)}{A \sinh \left(\Delta_{1} z\right)+B \cosh \left(\Delta_{1} z\right)}\right),
\end{aligned}
$$

where $\Delta_{1}=\sqrt{M} / 2, z=x+4 \sqrt{\mu} \omega t / 3$ and $A$ and $B$ are constants.

Case 2.2 For $M=\lambda^{2}-4 \mu<0$, we obtain the trigonometric function solution

$$
\begin{aligned}
u(t, x)= & (1+4 \beta \lambda) \sqrt{\frac{2 \sqrt{\mu} \omega}{\alpha}} \\
& \left(\frac{\lambda}{2}-\Delta_{2} \frac{A \sin \left(\Delta_{2} z\right)+B \cos \left(\Delta_{2} z\right)}{A \cos \left(\Delta_{2} z\right)+B \sin \left(\Delta_{2} z\right)}\right),
\end{aligned}
$$

where $\Delta_{2}=\sqrt{-M} / 2, z=x+4 \sqrt{\mu} \omega t / 3$ and $A$ and $B$ are constants.

Case 2.3 For $M=\lambda^{2}-4 \mu=0$, we obtain the rational function solution

$$
u(t, x)=(1+4 \beta \lambda) \sqrt{\frac{2 \sqrt{\mu} \omega}{\alpha}}\left(\frac{B}{B z+A}-\frac{\lambda}{2}\right),
$$

where $z=x+4 \sqrt{\mu} \omega t / 3$ and $A$ and $B$ are constants.

The solution profile of (2.24) for $\lambda=-0.09, A=0, B=$ $1, \mu=0.01, \omega=0.03, \alpha=1$ is presented in Figure 1, whereas the solution profile of (2.26) for $\lambda=-0.8, A=$ $0.4, B=3, \mu=0.2, \omega=0.01, \alpha=0.1$ is given in Figure 2 .

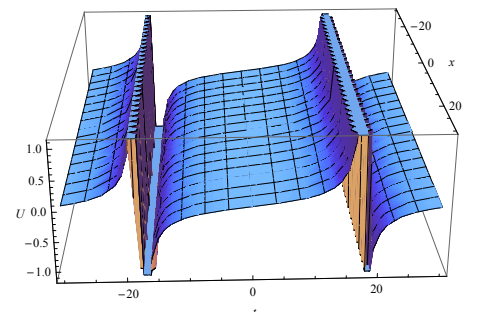

Figure 1: Profile of solution (2.24)

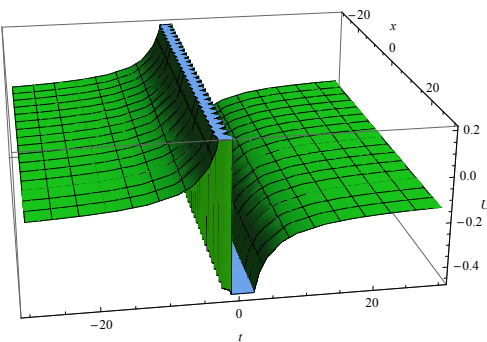

Figure 2: Profile of solution (2.26)

\section{Conservation laws of (1.5)}

In this section we derive conservation laws for the MEWBurgers equation (1.5) using the new conservation theorem due to Ibragimov [40, 41].

We begin by determining the adjoint equation of (1.5), namely

$$
F \equiv u_{t}+\alpha u^{2} u_{x}+\omega u_{x x}-\beta u_{t x x}=0,
$$

using the formula

$$
F^{\star} \equiv \frac{\delta}{\delta u}\left\{v\left(u_{t}+\alpha u^{2} u_{x}+\omega u_{x x}-\beta u_{t x x}\right)\right\}=0,
$$

where $\delta / \delta u$ is the Euler-Lagrange operator defined by

$$
\frac{\delta}{\delta u}=\frac{\partial}{\partial u}-D_{t} \frac{\partial}{\partial u_{t}}-D_{x} \frac{\partial}{\partial u_{x}}+D_{x}^{2} \frac{\partial}{\partial u_{x x}}-D_{t} D_{x}^{2} \frac{\partial}{\partial u_{t x x}}
$$

with the total differential operators $D_{t}$ and $D_{x}$ given by

$$
\begin{aligned}
D_{t}= & \frac{\partial}{\partial t}+u_{t} \frac{\partial}{\partial u}+v_{t} \frac{\partial}{\partial v}+u_{t t} \frac{\partial}{\partial u_{t}}+v_{t t} \frac{\partial}{\partial v_{t}} \\
& +u_{t x} \frac{\partial}{\partial u_{x}}+v_{t x} \frac{\partial}{\partial v_{x}}+\cdots, \\
D_{x}= & \frac{\partial}{\partial x}+u_{x} \frac{\partial}{\partial u}+v_{x} \frac{\partial}{\partial v}+u_{x x} \frac{\partial}{\partial u_{x}}+v_{x x} \frac{\partial}{\partial v_{x}} \\
& +u_{x t} \frac{\partial}{\partial u_{t}}+v_{x t} \frac{\partial}{\partial v_{t}} \cdots .
\end{aligned}
$$

Thus Equation (3.27) becomes

$$
F^{\star} \equiv v_{t}+\alpha u^{2} v_{x}-\omega v_{x x}-\beta v_{t x x}=0 \text {. }
$$

The MEW-Burgers equation (1.5) together with its adjoint equation (3.29) have the Lagrangian

$$
\mathcal{L}=v\left(u_{t}+\alpha u^{2} u_{x}+\omega u_{x x}-\beta u_{t x x}\right),
$$

which is equivalent to the second-order Lagrangian

$$
\mathcal{L}=v u_{t}+\alpha v u^{2} u_{x}+\omega v u_{x x}+\beta v_{t} u_{x x} .
$$

We recall that the MEW-Burgers equation (1.5) admits two translation symmetries $X_{1}=\partial / \partial t$ and $X_{2}=\partial / \partial x$. To obtain the conserved vectors corresponding to these two infinitesimal generators we use [40]

$$
\begin{aligned}
& C^{t}=\xi^{1} \mathcal{L}+W^{1} \frac{\partial \mathcal{L}}{\partial u_{t}}+W^{2} \frac{\partial \mathcal{L}}{\partial v_{t}}, \\
& C^{x}=\xi^{2} \mathcal{L}+W^{1}\left[\frac{\partial \mathcal{L}}{\partial u_{x}}-D_{x} \frac{\partial \mathcal{L}}{\partial u_{x x}}\right]+D_{x}\left(W^{1}\right) \frac{\partial \mathcal{L}}{\partial u_{x x}},
\end{aligned}
$$

where $W^{1}$ and $W^{2}$ are the Lie characteristic functions. Let us first consider the infinitesimal generator $X_{1}=\partial / \partial t$. It can be easily shown that the prolongation of the generator $X_{1}$ to the derivatives involved in the MEW-Burgers equation (1.5) has the form $\partial / \partial t$. In order to determine the value 
of $\lambda$ we use equation $X_{1}^{[3]}(F)=\lambda(F)$, which yields $\lambda=0$. Since $D_{t}\left(\xi^{1}\right)=0$, we obtain $\eta_{\star}=0$ and hence the operator admitted by the adjoint equation (3.29) is

$$
Y=\frac{\partial}{\partial t}
$$

We now use (3.34) to compute the Lie characteristic functions $W^{1}$ and $W^{2}$, which in this case are $W^{1}=-u_{t}$ and $W^{2}=-v_{t}$. Thus by using (3.32) and (3.33) the conserved vector for the system (1.5) and (3.29) corresponding to $X_{1}$ is

$$
\begin{aligned}
& C_{1}^{t}=\alpha v u^{2} u_{x}+\omega v u_{x x}, \\
& C_{1}^{x}=\omega v_{x} u_{t}+\beta v_{t x} u_{t}-\omega v u_{x t}-\alpha v u^{2} u_{t}-\beta u_{x t} v_{t} .
\end{aligned}
$$

Similarly, we compute the conserved vector corresponding to $X_{2}$. In this case $W^{1}=-u_{x}$ and $W^{2}=-v_{x}$ and the conserved vector thus rendered is

$$
\begin{aligned}
& C_{2}^{t}=-v u_{x}-\beta v_{x} u_{x x}, \\
& C_{2}^{x}=v u_{t}+\omega v_{x} u_{x}+\beta v_{t x} u_{x} .
\end{aligned}
$$

Remark: It should be noted that the time translation symmetry gives us the energy conservation law whereas the space translation symmetry provides us with the linear momentum conservation law.

\section{Conclusion}

In this paper we obtained travelling wave solutions of the MEW-Burgers equation (1.5). The two translation symmetries were used to reduce the MEW-Burgers equation to an ordinary differential equation. The $\left(G^{\prime} / G\right)$-expansion method was applied to the ordinary differential equation to obtain its solutions. Consequently travelling wave solutions were obtained for the MEW-Burgers equation. These solutions were hyperbolic, trigonometric and rational functions. Furthermore we derived two conservation laws using the new conservation theorem due to Ibragimov. These were the energy and the linear momentum conservation laws.

\section{References}

[1] Wazwaz A.M., El-Tantawy S.A., A new integrable $(3+1)$ - dimensional KdV-like model with its multiple-soliton solutions, Nonlin. Dyn., 2016, 83, 1529-1534.

[2] Wazwaz A.M., El-Tantawy S.A., Solving the $(3+1)$-dimensional KP-Boussinesq and BKP-Boussinesq equations by the simplified Hirota's method, Nonlin. Dyn., 2017, 88, 3017-3021.
[3] Wazwaz A.M., The tanh-coth method for solitons and kink solutions for nonlinear parabolic equations, Appl. Math. Comp., 2007, $188,1467-1475$.

[4] Wazwaz A.M., Exact solutions for the ZK-MEW equation by using the tanh and sine-cosine methods, J. Comp. Math., 2005, 82, 699-708.

[5] Kudryashov N.A., Exact solitary waves of the Fisher equation, Phys. Lett. A., 2005, 342, 99-106.

[6] Wang M., Zhou Y., Li Z., Application of a homogeneous balance method to exact solutions of nonlinear equations in mathematical physics, Phys. Lett. A, 1996, 216, 67-75.

[7] Ablowitz M.J., Clarkson P.A., Solitons, Nonlinear evolution equations and inverse scattering, 1991, Cambridge University Press, Cambridge.

[8] Hirota R., The direct method in soliton theory, 2004, Cambridge University Press, Cambridge.

[9] Wang M., Li X., Zhang J., The $\left(G^{\prime} / G\right)$ - expansion method and travellingwave solutions for linear evolution equations in mathematical physics, Phys. Lett. A, 2005, 24, 1257-1268.

[10] Mhlanga I.E., Khalique C.M., A study of a generalized BenneyLuke equation with time-dependent coefficients, Nonlin. Dyn., 2017, 90, 1535-1544.

[11] Baleanu D., Inc M., Yusuf A., Aliyu A.I., Traveling wave solutions and conservation laws for nonlinear evolution equation, J. Math. Phys., 2018, 59, 023506.

[12] Zhang Z., Jacobi elliptic function expansion method for the modi ed Korteweg-de Vries-Zakharov-Kuznetsov and the Hirota equations, Phys. Lett. A, 2001, 289, 69-74.

[13] Simbanefayi I., Khalique C.M., Travelling wave solutions and conservation laws for the Korteweg-de Vries-Bejamin-BonaMahony equation, Results in Physics, 2018, 8, 57-63

[14] Kudryashov N.A., One method for finding exact solutions of nonlinear differential equations, Comm. Nonlin. Sci. Numer. Simulat., 2012, 17, 2248-2253.

[15] Motsepa T., Khalique C.M., Conservation laws and solutions of a generalized coupled (2+1)-dimensional Burgers system, Comp. Math. Appl., 2017, 74, 1333-1339.

[16] Olver P.J., Applications of lie groups to differential equations, Springer-Verlag, New York, 1993.

[17] Ibragimov N.H. (Ed.), CRC Handbook of Lie group analysis of differential equations, 1994-1996, vol. 1-3, CRC Press, Boca Raton.

[18] Ibragimov N.H., Elementary Lie group analysis and ordinary differential equations, 1999, John Wiley and Sons, Chichester.

[19] Hydon P.E., Symmetry methods for differential equations, 2000, Cambridge, Cambridge University Press.

[20] Motsepa T., Khalique C.M., Gandarias M.L., Symmetry analysis and conservation laws of the Zoomeron equation, Symmetry, 2017, 9, 27.

[21] Motsepa T., Aziz T., Fatima A., Khalique C.M., Algebraic aspects of evolution partial differential equation arising in the study of constant elasticity of variance model from financial mathematics, Open Phys., 2018, 16, 31-36.

[22] Naz R., Mahomed F.M., Mason D.P., Comparison of different approaches to conservation laws for some partial differential equations in fluid mechanics, Appl. Math. Comp., 2008, 205, 212-230.

[23] Anco S.C., Bluman G., Direct construction method for conservation laws of partial differential equations. Part I: Examples of conservation law classifications, Eur. J. Pure Appl. Math., 2002, $13,545-566$. 
[24] Anco S.C., Bluman G., Direct construction method for conservation laws of partial differential equations. Part II: General treatment, Eur. J. Pure Appl. Math., 2002, 13, 567-585.

[25] De la Rosa R., Bruzón M.S., On the classical and nonclassical symmetries of a generalized Gardner equation, Appl. Math. Nonlin. Sci., 2016, 1, 263-272.

[26] Rosa M., Gandarias M.L., Multiplier method and exact solutions for a density dependent reaction-diffusion equation, Appl. Math. Nonlin. Sci., 2016, 1, 311-320.

[27] Gandarias M.L., Bruzón M.S., Conservation laws for a Boussinesq equation, Appl. Math. Nonlin. Sci., 2017, 2, 465-472.

[28] Motsepa T., Khalique C.M., On the conservation laws and solutions of a $(2+1)$ dimensional KdV-mKdV equation of mathematical physics, Open Phys., 2018, 16, 211-214.

[29] Leveque R.J., Numerical methods for conservation laws, 1992, Birkhäuser, Basel.

[30] Burgers J.M., Application of a model system to illustrate some 20 points of statistical theory of free turbulence, Royal Netherlands Acad. Sci., XLIII 1940, 1, 2-12.

[31] Morrison P.J., Meiss J.D., Cary J.R., Scattering of regularized long- wave solitary waves, Physica, 1984, 11D, 324-336.

[32] Zaki S.I., A least-squares finite element scheme for the EW equa tion, Comp. Meth. Appl. Mech. Eng., 2000, 189, 587-594.
[33] Zaki S.I., Solitary waves induced by the boundary forced EW equation, Comp. Meth. Appl. Mech. Eng., 2001, 190, 4881-4887.

[34] Arora R., Siddiqui Md.J., Singh V.P., Solutions of invscid Burgers' and equal width wave equations by RDTM, International J. Appl. Phys. Math., 2012, 2, 212-214..

[35] Zaki S.I., Solitarywave interactions for the modified equalwidth equation, Comp. Phys. Comm., 2000, 126, 219-231.

[36] Wazwaz A.M., The tanh and sine-cosine methods for a reliable treatment of the modified equalwidth equation and its variants, Comm.. Nonlin. Sci. Numer. Simul., 2006, 11, 148-160.

[37] Hassan H.N., An accurate numerical solution for the modified equal width wave equation using the Fourier pseudo-spectral method, J. Appl. Math. Phys., 2016, 4, 1054-1067.

[38] Evans D.J., Raslan K.R., Solitary waves for the generalized equal width (GEW) equation, Int. J. Comp. Math., 20005, 82, 445-455.

[39] Saha A., Bifurcation, periodic and chaotic motions of the modified equal width-Burgers (MEW-Burgers) equation with external periodic perturbation, Nonlin. Dyn., 2007, 87, 2193-2201.

[40] Ibragimov N.H., A new conservation theorem, J. Math. Anal. Appl., 2007, 333, 311-328.

[41] Mothibi D.M., Khalique C.M., Conservation laws and exact solutions of a generalized Zakharov-Kuznetsov equation, Symmetry, 2015, 7, 949-961. 